\title{
Design of Inverter Side of Modular Grid Simulator Based on Proportional integral-Quasi-proportional resonance Control
}

\author{
Ren Huai Xin ${ }^{1}$, Xie Yuan ${ }^{1}$, and Zhang Kai ${ }^{1}$ \\ ${ }^{1}$ Shanghai Dianji University School of Electrical Engineering, Pudong New Area201306, Shanghai, China
}

\begin{abstract}
The tracking of a given voltage in the traditional double closed-loop proportional integral control in the current power grid simulator has problems such as static difference, delay and oscillation. It is proposed that the voltage outer loop and current inner loop of the inverter side of the power grid simulator adopt proportional integral and quasi-proportional resonance control respectively, and the topology of the inverter adopts a cascaded modular design to establish a single-phase inverter model. Compared with the traditional double closed-loop proportional-integral control, it is verified that the proportional-integral-quasiproportional resonant controller can effectively improve the system's ability to track the command voltage and the stability of the output voltage.
\end{abstract}

\section{Introduction}

In the process of researching wind power generation systems, it is essential to test and study the operating characteristics of the wind power generation system when the grid fails. In actual grid connection, it can provide a certain amount of active or reactive power when the grid fails. Support ${ }^{[1]}$. Due to the randomness and uncontrollability of power grid faults, it is of great significance to study power grid simulators that can simulate various fault situations of the power grid.

This paper proposes a topological structure of a combined inverter. At present, there are many researches on the rectifier side, and this article focuses on the inverter side. By analyzing the topology and principle of the combined inverter, Carrier Phace-Shift Modulation (CPSM), which is the most commonly used and has strong dynamic regulation ability, is adopted to effectively reduce harmonics at a lower switching frequency ${ }^{[3]}$. Based on the Matlab/Simulink software simulation platform, a simulation model of the voltage source combined inverter is built, the experimental results are analyzed, and some help is provided for the control research of the combined inverter.

\section{Topological structure of the combined inverter}

The overall structure of the power grid simulator is shown in Figure 1, which is mainly divided into two parts: the rectifier side and the inverter side. Among them, the rectifier side DC power supply replaces the rectifier part, and the inverter side adopts a combined inverter structure.

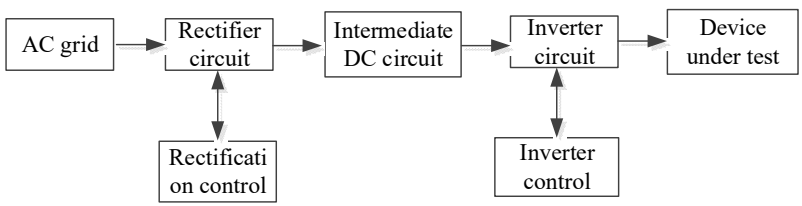

Fig 1 Schematic diagram of the overall structure of the power grid simulator

Taking into account the needs of research, start with the two-module combined inverter. As shown in Figure 2, this article selects the sub-IGBT in the two-module cascaded inverter.

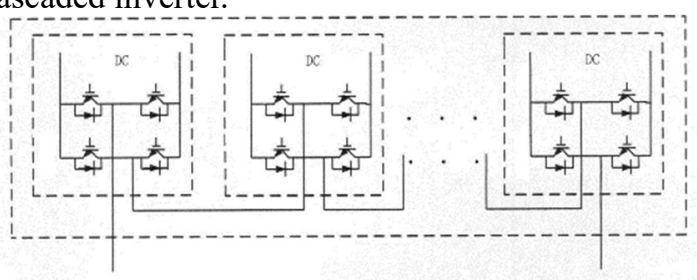

Fig 2 Modular inverter

The two-module cascade inverter is improved on the basis of the original single-phase full bridge and used as a sub-IGBT module. Each module maintains the original DC power supply to provide power. Between the two modules, connect the negative output of the previous module to the positive pole of the next stage, and combine the negative output of the next stage and the positive pole of the previous stage into a new one. The sub-IGBT module is used.

The control strategy in this paper adopts phase-shifting technology, and adopts double-loop control of voltage and current for the whole system in the article. Among them, the outer loop voltage loop adopts PI control, and the inner loop current loop adopts PR control. Among them, the current inner loop increases the bandwidth of the system,

\footnotetext{
* Corresponding author: rhxsdju@163.com xiey@sdju.edu.cn
} 
improves the dynamic response of the system, strengthens the ability to adapt to disturbances, and reduces the harmonic content.

The phase-shifting technology is to adopt phaseshifting control on the control carrier of the bridge arm corresponding to the different inverters in the cascade. In turn, the modulated output waveform of each cascaded inverter has a superimposing effect, making it closer to a sine wave while ensuring the same fundamental wave ${ }^{[4]}$. Figure 3 is a schematic diagram of a phase shifting technique using SPWM modulation.

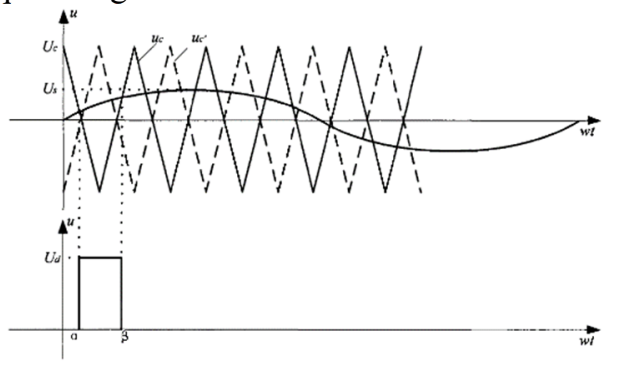

Fig 3 SPWM phase-shift modulation principle diagram

The triangular carrier $U_{C}$ in the figure is:

$$
\begin{aligned}
& -\frac{2 U_{c}}{\pi} \omega_{c} t+U_{c}\left(0 \leq \omega_{c} t \leq \pi\right) \\
& \frac{2 U_{c}}{\pi} \omega_{c} t-3 U_{c}\left(\pi \leq \omega_{c} t \leq 2 \pi\right)
\end{aligned}
$$

In the figure, it is assumed that the modulating wave $U_{S} \sin \left(\omega_{S} t\right)$ is. The intersection of the modulating wave and the carrier is calculated as:

$$
\begin{aligned}
& \alpha=\theta-\frac{\pi}{2}\left(M \sin \left(\omega_{s} t\right)-1\right) \\
& \beta=\theta+\frac{\pi}{2}\left(M \sin \left(\omega_{s} t\right)+3\right)
\end{aligned}
$$

Where $\theta$ is the carrier phase shift angle and $M=U_{s} / U_{c}$ is the modulation ratio. According to formula (2), the output pulse wave after modulation is expanded according to the Fourier series, and the output voltage of the left bridge arm of the single-phase inverter is:

$$
\begin{aligned}
& U_{+}=U_{d} M \sin \left(\omega_{s} t\right) \\
& +\sum_{n=1}^{\infty} \frac{4 U_{d}}{n \pi} \cos (n \theta+n \pi) \sin \left(\frac{n \pi}{2} M \sin \left(\omega_{s} t\right)+\frac{n \pi}{2}\right) \cos \left(n \omega_{c} t\right) \\
& +\sum_{n=1}^{\infty} \frac{4 U_{d}}{n \pi} \sin (n \theta+n \pi) \sin \left(\frac{n \pi}{2} M \sin \left(\omega_{s} t\right)+\frac{n \pi}{2}\right) \sin \left(n \omega_{c} t\right)
\end{aligned}
$$

In the same way, the output voltage of the right bridge arm is (modulation wave reverse):

$$
U_{+}=-U_{d} M \sin \left(\omega_{s} t\right)
$$$$
+\sum_{n=1}^{\infty} \frac{4 U_{d}}{n \pi} \cos (n \theta+n \pi) \sin \left(-\frac{n \pi}{2} M \sin \left(\omega_{s} t\right)+\frac{n \pi}{2}\right) \cos \left(n \omega_{c} t\right)
$$$$
+\sum_{n=1}^{\infty} \frac{4 U_{d}}{n \pi} \sin (n \theta+n \pi) \sin \left(-\frac{n \pi}{2} M \sin \left(\omega_{s} t\right)+\frac{n \pi}{2}\right) \sin \left(n \omega_{c} t\right)
$$

Suppose the carrier phase shift angle of another singlephase inverter $U_{c}{ }^{\prime}$ is $\theta$. In the same way, $U_{+}{ }^{\prime}$ and $U_{-}{ }^{\prime}$ can be calculated. Finally, the output voltages of the two single-phase cascaded inverters are $U=\left(U_{+}-U_{-}\right)+\left(U_{+}^{\prime}-U_{-}^{\prime}\right)$

$=4 U_{d} M \sin \left(\omega_{s} t\right)+\sum_{n=1}^{\infty} \frac{8 U_{d}}{n \pi} \cos \frac{n \pi}{2} \sum_{k=1,3, \ldots}^{\infty}(-1)^{\frac{k-1}{2}} J_{k}\left(\frac{M n \pi}{2}\right)$

$\left\{[\cos (n \theta+n \pi)+\cos (n \theta+n \pi)] \sin \left(n k_{c} \pm k\right) \omega_{s} t[\sin (n \theta+n \pi)+\sin (n \theta+n \pi)] \cos \left(n k_{c} \pm k\right) \omega_{s} t\right\}$

Where $K_{c}=\omega_{s} / \omega_{c}$ is the carrier ratio. Analytical formula (5) When $\theta=\pi / 2$ the lowest harmonic order of the output voltage reaches $4 n K_{c} \pm 1$, the lowest harmonic order at other angles are $2 n K_{c} \pm 1$ all. Therefore, when designing the phase shift angle of the inverter $\pi / 2$, the harmonic content of the system is reduced.

\section{Inverter side design}

\subsection{Filter parameter design}

The main function of the inverter's output filter is to filter out the harmonics of the switching frequency and its adjacent frequency bands, as shown in the figure. This article considers the parameter design of the filter from the characteristics of the second-order LC filter.

In the figure, ignoring the inductor resistance and line impedance, the transfer function between the output voltage of the filter and the output voltage of the inverter bridge at this time:

$$
\begin{aligned}
& G(s)=V_{0}(s) / V_{i}(s) \\
& =\frac{\frac{1}{L C}}{s^{2}+\frac{1}{R C} s+\frac{1}{L C}}=\frac{\omega_{n}{ }^{2}}{s^{2}+2 \xi \omega_{n} s+\omega_{n}{ }^{2}}
\end{aligned}
$$

Where $\omega_{n}=1 / L C$ is the undamped natural oscillation angular frequency and $\xi=\frac{1}{2 R} \sqrt{L / C}$ damping ratio. This is a typical second-order oscillation system, and its frequency characteristic is

$$
\begin{aligned}
& G(j \omega)=\frac{\omega_{n}{ }^{2}}{\omega_{n}{ }^{2}-\omega^{2}+j 2 \xi \omega_{n} \omega} \\
& =\frac{1}{1-\left(\frac{\omega}{\omega_{n}}\right)^{2}+j 2 \xi \frac{\omega}{\omega_{n}}}=A(\omega) e^{j \varphi(\omega)}
\end{aligned}
$$

Where

$$
\begin{aligned}
& A(\omega)=\frac{1}{\sqrt{\left[1-\left(\frac{\omega}{\omega_{n}}\right)^{2}\right]+\left(2 \xi \frac{\omega}{\omega_{n}}\right)^{2}}} \\
& \varphi(\omega)=-\operatorname{arctg}\left[\frac{2 \xi \frac{\omega}{\omega_{n}}}{1-\left(\frac{\omega}{\omega_{n}}\right)^{2}}\right]
\end{aligned}
$$

According to the above formula (7), the logarithmic amplitude-frequency characteristic can be easily obtained:

$$
L(\omega)=20 \lg A(\omega)=-20 \lg \sqrt{\left[1-\left(\frac{\omega}{\omega_{n}}\right)^{2}\right]^{2}+\left(2 \xi \frac{\omega}{\omega_{n}}\right)^{2}}
$$


In the low frequency band, instantaneous $\omega \ll \omega_{n}$, $A(\omega) \approx 1, L(\omega) \approx 0$ can be obtained; in the high frequency band, instantaneous $\omega \gg \omega_{n}, \quad A(\omega) \approx 1 / L C \omega^{2}$

$L(\omega) \approx-40 \lg (\sqrt{L C} \omega)$, can be obtained. It can be concluded that the low-frequency asymptote is a zerodecibel horizontal line, and the high-frequency asymptote is a straight line with a slope of $-40 \mathrm{~dB}$. The handover frequency at the intersection of these two lines is $\omega_{1}=\omega_{n}$. Near the handover frequency, there is a certain error between the amplitude-frequency characteristic and the asymptote, and its value depends on the value of the damping ratio $\xi$. The smaller the damping ratio, the greater the error. When the damping ratio $\xi$ is less than 0.707, the linear peak is deleted in the logarithmic amplitude-frequency characteristic. Based on the abovementioned combined literature ${ }^{[5]}$, the cut-off frequency of the $L C$ filter is $1 / 10$ taken as the switching frequency. In this paper, the switching frequency is $f_{s}=40 \mathrm{kHz}$, the cut-off frequency is $f_{n}=\frac{1}{10} f_{s}=4 \mathrm{kHz}, \quad L=0.636695 \mathrm{mH}, C=2.38545 \mu \mathrm{F}$

\subsection{Inverter side control design}

The inverter side of the power grid simulator adopts dualloop control of voltage outer loop and current inner loop. The current inner loop increases the bandwidth of the system, improves the dynamic response of the system, and strengthens the ability to adapt to disturbances. Using the inductor current as the inner loop feedback can effectively improve the response speed to the load voltage, but it cannot effectively control the load current. Therefore, in order to improve the anti-disturbance capability of the system, this article adds feedforward compensation for load disturbance on the basis of the original inductor current to improve this effect.

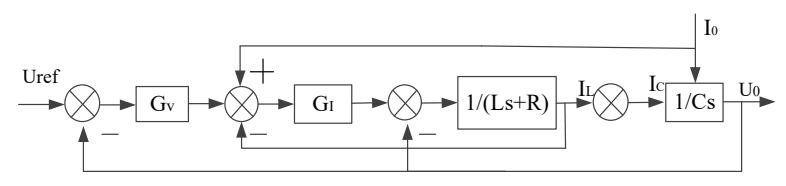

Fig 4 Inverter control block diagram

\subsection{Design of proportional-integral-quasi- proportional resonant controller}

Compared with the traditional proportional-integral (PI) double-loop control that cannot realize the non-static control of the AC signal, the ideal proportional resonance control (PR) has infinite gain at the fundamental frequency, and its ideal transfer function is:

$$
G_{p r}(\mathrm{~s})=\mathrm{K}_{p}+\frac{K_{R} s}{s^{2}+\omega_{0}{ }^{2}}
$$

Where $K_{p}$ is the proportional coefficient and $K_{R}$ is the resonant link gain. Compared with PR control, quasiproportional control (QPR) has the characteristics of high gain at the fundamental frequency point, and can also expand the bandwidth of high gain. Even if the frequency is slightly shifted, it can still achieve a good tracking effect. The transfer function of the quasi-proportional resonant controller is:

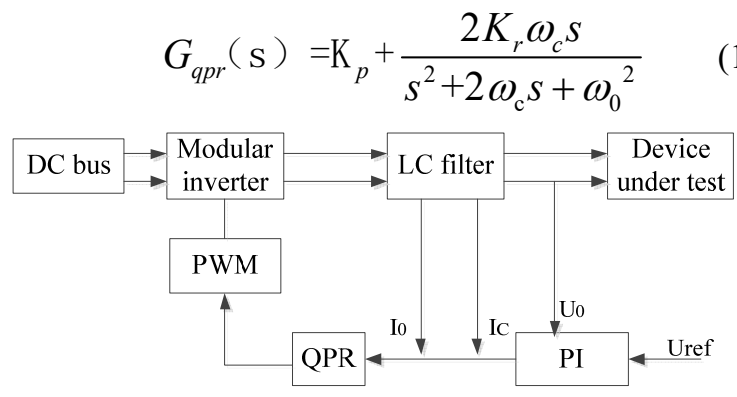

Fig 5 Control schematic diagram

Suppose the voltage and current regulators are respectively:

$$
\begin{aligned}
& G_{v}(s)=K_{p 1}+\frac{K_{i 1}}{s} \\
& G_{i}(s)=K_{p 2}+\frac{2 K_{R 2} \omega_{c} s}{s^{2}+2 \omega_{c} s+\omega_{0}^{2}}
\end{aligned}
$$

The control principle diagram is as shown in the figure above, where $U_{0}$ is the inverter side output voltage, $i_{0}$ is the load current output by the inverter side, and $i_{1}$ is the inductor current. The voltage output from the inverter side $U_{0}$ is compared with the reference voltage $U_{r e f}$, and the error signal obtained is used as the reference current of the inner loop, the inductor current and the load feedforward current $i_{0}$, and finally the PWM signal is generated, and finally the modulation signal is generated by the phase shift technology.

\section{Inverter side simulation of grid simulator}

In order to verify the feasibility of the proposed model and control strategy, a simulation model of the inverter side was built based on MATLAB/SIMULINK. The selected parameters were: $U_{d c}=600 \mathrm{~V}$, the effective value of the load side voltage $311 \mathrm{~V}$, $L=0.636695 \mathrm{mH}, \mathrm{C}=2.38545 \mu \mathrm{F}, f_{k}=40 \mathrm{kHz}$

Figure 5 hows the PI-QPR dual-loop control System Block Diagram. The current inner loop control adopts PR control and the voltage outer loop control adopts PI control, which $U_{r e f}$ is a given voltage waveform, and the output terminal is the output voltage of the inverter side, that is, the voltage across the device under test. The outer loop control parameters $K_{p}=0.08, K_{i}=5$,inner loop parameters $K_{r}=1000 \pi, \omega_{c}=31.4$, 
$\omega_{0}=1000$ in the control circuit.

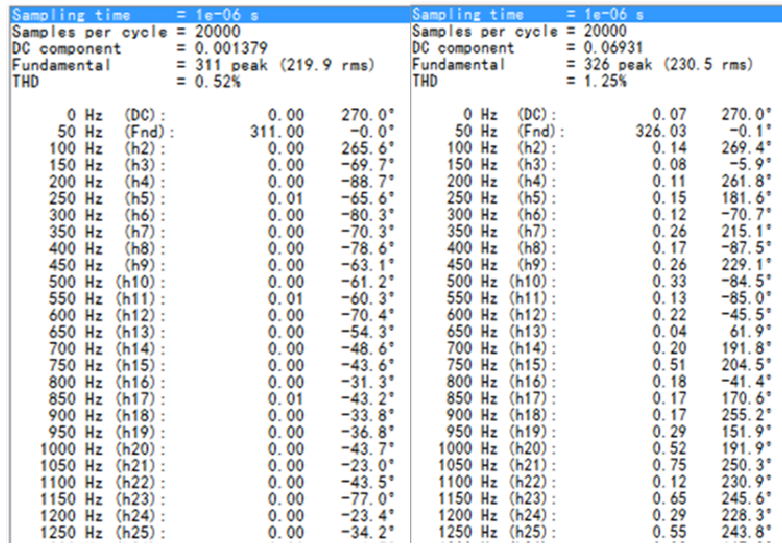

Fig 6 PI-QPR (left) and PI-PI (right) output voltage Fourier analysis comparison

From Fig 6, the comparison of the FFT analysis chart shows that the harmonic characteristics of the output voltage controlled by PI-QPR are better, and its THD is $0.52 \%$, which far meets the national standard GBT14549$935 \%$. Reasonably designed phase shifting and double closed-loop control can well regulate and control the cascaded inverters. In the case of system differences, lower THD voltage waveforms can be obtained, and the system can be improved under the premise of ensuring accuracy. The output voltage.

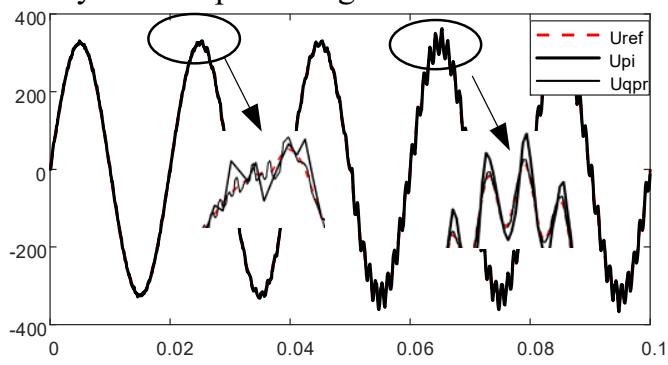

Fig 7 Simulation comparsion chart

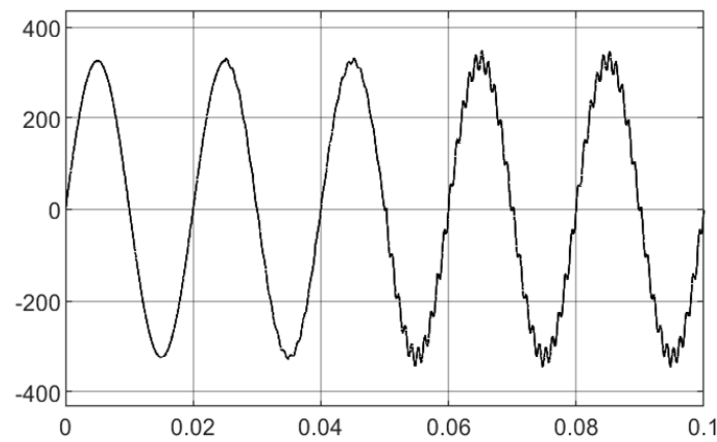

Fig 8 Voltage follower of PI-QPR control

\section{Conclusion}

It can be found from Figure 6 that the voltage output distortion rate based on proportional-quasi-proportional resonance control is lower than the voltage output distortion rate of dual proportional-integral control, which is increased by 0.73 . From the simulation comparison in Figure 7, it is clear that the Compared with the traditional dual PI control, the control strategy can track the reference voltage better, and the fluctuation is smaller. It can be found from Figure 8 that the first cycle is the $50 \mathrm{~Hz}$ fundamental wave tracking effect, the second cycle adds a harmonic effect with an amplitude of 5, and the third cycle and the fourth cycle have amplitudes of 10 and 20 in sequence. The doubling harmonics. It can be clearly seen from Figures 7 and 8 that both can effectively track, and the effect is better than traditional control. Therefore, the topology and control used in the article can track the reference signal well, verifying the effectiveness of the strategy in the article.

\section{References}

1. Han Rong, $\mathrm{Xu}$ Qianming, Ding Hongqi, et al. Modular multi-level mid-high voltage power grid simulator and its control[J]. Transactions of the China Electrotechnical Society, 2018, 33(S1): 165-175。

2. R. Lohde and F. W. Fuchs" Laboratory type PWM grid emulator for generating disturbed voltages for generating disturbed voltages for testing grid connected devices" Power Electronics and Applications 2009.EPE '09. 13th European Conference on pp. 1-9 2009.

3. Zhu Hong, Zhang Xing, Li Fei, et al. Research on high-power power grid simulator system[J]. Journal of Power Supply, 2017, 15(3): 163-167.

4. Ke Yichen, Chen Guochu, Xu Yiqing, et al. Design of inverter side of power grid simulator based on improved sliding mode control[J/OL]. Electrical measurement and instrumentation: 1-7[2020-07-06].

5. He Liang, Wang Jinsong. Three-phase PWM inverter output LC filter design method[J]. Electric Drive, 2013, 43(12): 33-36. 